\title{
SUFFICIENT AND NECESSARY CONDITION FOR THE PERMANENCE OF PERIODIC PREDATOR-PREY SYSTEM
}

\author{
JINGAN CUI and XINYU SONG
}

Received 22 January 2003

\begin{abstract}
We consider the permanence of a periodic predator-prey system, where the prey disperse in a two-patch environment. We assume the Volterra within-patch dynamics and provide a sufficient and necessary condition to guarantee the predator and prey species to be permanent by using the techniques of inequality analysis. Our work improves previous relevant results.
\end{abstract}

2000 Mathematics Subject Classification: 92D25, 34C60.

1. Introduction. Dispersal predator-prey systems described by autonomous ordinary differential equations have long played an important role in population biology (see $[1,2,3,4,5,7,8,9,10,11,12,13,14,15,18,19,20,21,22,23,24]$ and the references cited therein). Recently, Lou and Ma [15] studied the following predator-prey system in two-patch environment:

$$
\begin{aligned}
\dot{x}_{1} & =x_{1}\left(b_{1}-a_{1} x_{1}-c_{1} y\right)+D\left(x_{2}-x_{1}\right), \\
\dot{x}_{2} & =x_{2}\left(b_{2}-a_{2} x_{2}\right)+D\left(x_{1}-x_{2}\right), \\
\dot{y} & =y\left(-d+c_{2} x_{1}-l y\right),
\end{aligned}
$$

where $x_{i}(t)$ represents the prey population in the $i$ th patch, $i=1,2$, at time $t \geq 0$, $y(t)$ stands for the predator population in patch 1 at time $t \geq 0$; coefficients $a_{i}, b_{i}, c_{i}$ $(i=1,2), d, l$, and $D$ are all positive constants. They proved that

$$
-d+c_{2} x_{1}^{*}(D)>0
$$

is a necessary and sufficient condition of the strong persistence of system (1.1), where $\left(x_{1}^{*}(D), x_{2}^{*}(D)\right)$ is the globally asymptotically stable equilibrium of the following system:

$$
\begin{aligned}
& \dot{x}_{1}=x_{1}\left(b_{1}-a_{1} x_{1}\right)+D\left(x_{2}-x_{1}\right), \\
& \dot{x}_{2}=x_{2}\left(b_{2}-a_{2} x_{2}\right)+D\left(x_{1}-x_{2}\right) .
\end{aligned}
$$


Considering realistic models often requires the effects of the changing environment; we naturally expect that a similar condition should be selected for the permanence of the corresponding periodic predator-prey system,

$$
\begin{aligned}
\dot{x}_{1} & =x_{1}\left[b_{1}(t)-a_{1}(t) x_{1}-c_{1}(t) y\right]+D(t)\left(x_{2}-x_{1}\right), \\
\dot{x}_{2} & =x_{2}\left[b_{2}(t)-a_{2}(t) x_{2}\right]+D(t)\left(x_{1}-x_{2}\right), \\
\dot{y} & =y\left[-d(t)+c_{2}(t) x_{1}-l(t) y\right],
\end{aligned}
$$

under the assumptions that the functions $a_{i}(t), b_{i}(t), c_{i}(t)(i=1,2), D(t), d(t)$, and $l(t)$ are all positive, $\omega$-periodic, and continuous for $t \geq 0$.

Existing results on the permanence of system (1.4) have largely been restricted to some roughly sufficient conditions due to the increased complexity of global analysis for the nonautonomous systems (cf. Song and Chen [18]). The present paper provides a necessary and sufficient condition of the permanence of system (1.4) and removes some unnecessary conditions in [18].

The organization of this paper is as follows. In Section 2, we agree on some notations, give some definitions, and state three lemmas which will be essential to our proofs. In Section 3, by introducing the techniques found in [21], we obtain the necessary and sufficient condition which guarantees that system (1.4) is permanent.

2. Notations, definitions, and preliminaries. In this section, we introduce some definitions and notations and state some results which will be useful in subsequent sections. Let $C$ denote the space of all bounded continuous functions $f: \mathbb{R} \rightarrow \mathbb{R}, C_{+}^{0}$ the set of nonnegative $f \in C$, and $C_{+}$the set of all $f \in C$ such that $f$ is bounded below by a positive constant. Given $f \in C$, we denote

$$
f^{M}=\sup _{t \geq 0} f(t), \quad f^{L}=\inf _{t \geq 0} f(t),
$$

and define the lower average $A_{L}(f)$ and upper average $A_{M}(f)$ of $f$ by

$$
\begin{aligned}
& A_{L}(f)=\lim _{r \rightarrow \infty} \inf _{t-s \geq r}(t-s)^{-1} \int_{s}^{t} f(\tau) d \tau, \\
& A_{M}(f)=\lim _{r \rightarrow \infty} \sup _{t-s \geq r}(t-s)^{-1} \int_{s}^{t} f(\tau) d \tau,
\end{aligned}
$$

respectively. If $f \in C$ is $\omega$-periodic, we define the average $A_{\omega}(f)$ of $f$ on the time interval $[0, \omega]$ by

$$
A_{\omega}(f)=\omega^{-1} \int_{0}^{\omega} f(t) d t
$$

DEFINITION 2.1. The system of differential equations

$$
\dot{x}=F(t, x), \quad x \in \mathbb{R}^{n},
$$

is said to be permanent if there exists a compact set $K$ in the interior of $\mathbb{R}_{+}^{n}=\left\{\left(x_{1}, x_{2}, \ldots\right.\right.$, $\left.\left.x_{n}\right) \in \mathbb{R}^{n}: x_{i} \geq 0, i=1,2, \ldots, n\right\}$ such that all solutions starting in the interior of $\mathbb{R}_{+}^{n}$ 
ultimately enter and remain in $K$. The system is said to be strongly persistent if

$$
\liminf _{t \rightarrow \infty} x_{i}(t)>0, \quad i=1,2, \ldots, n,
$$

hold for all solutions $x(t)=\left(x_{1}(t), x_{2}(t), \ldots, x_{n}(t)\right)$ starting in the interior of $\mathbb{R}_{+}^{n}$.

DEFINITION 2.2. The system of differential equations

$$
\dot{x}=F(t, x), \quad(t, x) \in \mathbb{R} \times \mathbb{R}^{n},
$$

is said to be cooperative if the off-diagonal elements of $D_{x} F(t, x)$ are nonnegative and competitive if the off-diagonal elements are nonpositive, where $D_{x} F(t, x)$ is the $n \times n$ matrix derivative of $F$ with respect to $x$.

LEMMA 2.3 [17]. Let $x(t)$ and $y(t)$ be solution of

$$
\dot{x}=F(t, x), \quad \dot{y}=G(t, y),
$$

respectively, where both systems are assumed to have the uniqueness property for initial value problems. Assume both $x(t)$ and $y(t)$ belong to a domain $D \subset \mathbb{R}^{n}$ for $\left[t_{0}, t_{1}\right]$, in which one of the two systems is cooperative and

$$
F(t, z) \leq G(t, z), \quad(t, z) \in\left[t_{0}, t_{1}\right] \times D .
$$

If $x\left(t_{0}\right) \leq y\left(t_{0}\right)$, then $x\left(t_{1}\right) \leq y\left(t_{1}\right)$. If $F=G$ and $x\left(t_{0}\right)<y\left(t_{0}\right)$, then $x\left(t_{1}\right)<y\left(t_{1}\right)$.

To prove the permanence of the species in (1.4), we need the information on the periodic logistic models with and without dispersal.

LEMMA 2.4 [25]. The problem

$$
\dot{x}=x[b(t)-a(t) x], \quad x \in C_{+}
$$

has exactly one canonical solution $U$ if $a \in C_{+}, b \in C$, and $A_{L}(b)>0$. Moreover, the following properties hold:

(a) $U$ is $\omega$-periodic (almost periodic) if $a, b$ are $\omega$-periodic (almost periodic);

(b) $U$ is constant if $b / a$ is constant. In this case, $U=b / a$;

(c) $u(t)-U(t) \rightarrow 0$ as $t \rightarrow \infty$, for any positive solution $u(t)$ of (2.9);

(d) $(b / a)^{L} \leq U \leq(b / a)^{M}$.

For the dispersal logistic equations

$$
\begin{aligned}
& \dot{x}_{1}=x_{1}\left[b_{1}(t)-a_{1}(t) x_{1}\right]+D(t)\left(x_{2}-x_{1}\right), \\
& \dot{x}_{2}=x_{2}\left[b_{2}(t)-a_{2}(t) x_{2}\right]+D(t)\left(x_{1}-x_{2}\right),
\end{aligned}
$$

we have the following result.

LEMMA 2.5 [16]. Suppose that $b_{i}(t), a_{i}(t)(i=1,2)$, and $D(t)$ are all positive and $\omega$-periodic functions; then (2.10) has a positive and $\omega$-periodic solution $\left(x_{1}^{*}(t), x_{2}^{*}(t)\right)$ which is globally asymptotically stable. 


\section{Necessary and sufficient condition of permanence in (1.4)}

THEOREM 3.1. System (1.4) is permanent if and only if

$$
A_{\omega}\left[-d(t)+c_{2}(t) x_{1}^{*}(t)\right]>0,
$$

where $\left(x_{1}^{*}(t), x_{2}^{*}(t)\right)$ is the globally asymptotically stable periodic solution of (2.10).

To prove this theorem, we need several propositions. In the rest of this paper, we denote by $\left(x_{1}(t), x_{2}(t), y(t)\right)$ any solution of (1.4) with positive initial condition.

Proposition 3.2. There exist positive constants $M_{x}$ and $M_{y}$ such that

$$
\lim _{t \rightarrow \infty} \sup x_{i}(t) \leq M_{x}, \quad \lim _{t \rightarrow \infty} \sup y(t) \leq M_{y}, \quad i=1,2 .
$$

Proof. Obviously, $\mathbb{R}_{+}^{3}$ is a positively invariant set of (1.4). Given any positive solution $\left(x_{1}(t), x_{2}(t), y(t)\right)$ of (1.4), we have

$$
\dot{x}_{i} \leq x_{i}\left[b_{i}(t)-a_{i}(t) x_{i}\right]+D(t)\left(x_{j}-x_{i}\right), \quad i=1,2, j \neq i ;
$$

on the other hand, the auxiliary equations

$$
\dot{u}_{i}=u_{i}\left[b_{i}(t)-a_{i}(t) u_{i}\right]+D(t)\left(u_{j}-u_{i}\right), \quad i=1,2, j \neq i,
$$

have a unique globally asymptotically stable positive $\omega$-periodic solution $\left(x_{1}^{*}(t)\right.$, $\left.x_{2}^{*}(t)\right)$. Let $\left(u_{1}(t), u_{2}(t)\right)$ be the solution of (3.4) with $u_{i}(0)=x_{i}(0)$. By Lemma 2.3, we have

$$
x_{i}(t) \leq u_{i}(t), \quad i=1,2 \text {, for } t \geq 0 \text {. }
$$

Moreover, from the global stability of $\left(x_{1}^{*}(t), x_{2}^{*}(t)\right)$, for every given $\varepsilon>0$, there exists $T_{0}>0$ such that

$$
u_{i}(t)<x_{i}^{*}(t)+\varepsilon \text { for } t>T_{0}
$$

hence

$$
x_{i}(t)<x_{i}^{*}(t)+\varepsilon, \quad i=1,2 \text {, for } t>T_{0} .
$$

In addition, for $t \geq T_{0}$, we have

$$
\dot{y} \leq y\left[-d(t)+c_{2}(t)\left(x_{1}^{*}(t)+\varepsilon\right)-l(t) y\right] .
$$

By (3.1), and Lemmas 2.3 and 2.4, there exists $T_{1}>T_{0}$ such that

$$
y(t)<y^{*}(t)+\varepsilon \text { for } t>T_{1},
$$

where $y^{*}(t)$ is the positive and globally asymptotically stable $\omega$-periodic solution of the auxiliary logistic equation

$$
\dot{v}=v\left[-d(t)+c_{2}(t)\left(x_{1}^{*}(t)+\varepsilon\right)-l(t) v\right] .
$$


Denote $M_{x}=\max _{0 \leq t \leq \omega}\left\{x_{i}^{*}(t)+\varepsilon: i=1,2\right\}$ and $M_{y}=\max _{0 \leq t \leq \omega}\left\{y^{*}(t)+\varepsilon\right\}$; then (3.2) holds for system (1.4).

Proposition 3.3. There exists a positive constant $\eta_{x}$ such that

$$
\lim _{t \rightarrow \infty} \sup x_{1}(t) \geq \eta_{x}
$$

Proof. Suppose that (3.11) is not true; then there is a sequence $\left\{z_{m}\right\} \subset \mathbb{R}_{+}^{3}$ such that

$$
\lim _{t \rightarrow \infty} \sup x_{1}\left(t, z_{m}\right)<\frac{1}{m}, \quad m=1,2, \ldots
$$

where $\left(x_{1}\left(t, z_{m}\right), x_{2}\left(t, z_{m}\right), y\left(t, z_{m}\right)\right)$ is the solution of (1.4) with initial values $\left(x_{1}\left(0, z_{m}\right), x_{2}\left(0, z_{m}\right), y\left(0, z_{m}\right)\right)=z_{m}$. Choosing sufficiently small positive constants $\varepsilon_{x}$ and $\varepsilon_{y}$ such that $\varepsilon_{x}<1, \varepsilon_{y}<1$, and

$$
\begin{gathered}
A_{\omega}\left(-d(t)+c_{2}(t) \varepsilon_{x}\right)<0, \\
A_{\omega}\left(\phi_{\varepsilon}(t)\right)>0,
\end{gathered}
$$

where $\phi_{\varepsilon}(t)=\min \left\{b_{1}(t)-c_{1}(t) \varepsilon_{y} \exp (\alpha \omega)-a_{1}(t) \varepsilon_{x}, b_{2}(t)-a_{2}(t) \varepsilon_{x}\right\}, \quad \alpha=$ $\max _{0 \leq t \leq \omega}\left\{|d(t)|+c_{2}(t)+l(t)\right\}$. By (3.12), for the given $\varepsilon_{x}>0$, there exists a positive integer $N_{0}$ such that

$$
\limsup _{t \rightarrow \infty} x_{1}\left(t, z_{m}\right)<\frac{1}{m}<\varepsilon_{x}
$$

for $m>N_{0}$. In the rest of this proof, we always assume that $m>N_{0}$. The above inequality implies that there exists $\tau_{1}^{(m)}>0$ such that

$$
x_{1}\left(t, z_{m}\right)<\varepsilon_{x}
$$

for $t \geq \tau_{1}^{(m)}$, and further

$$
\dot{y}\left(t, z_{m}\right) \leq y\left(t, z_{m}\right)\left[-d(t)+c_{2}(t) \varepsilon_{x}-l(t) y\left(t, z_{m}\right)\right]
$$

for $t \geq \tau_{1}^{(m)}$. By (3.13), any solution $v(t)$ of the equation

$$
\dot{v}=v\left[-d(t)+c_{2}(t) \varepsilon_{x}-l(t) v\right]
$$

with positive initial condition satisfies

$$
\lim _{t \rightarrow \infty} v(t)=0
$$

By Lemma 2.3, we have

$$
\lim _{t \rightarrow \infty} y\left(t, z_{m}\right)=0
$$


Therefore, there is a $\boldsymbol{\tau}_{2}^{(m)}>\boldsymbol{\tau}_{1}^{(m)}$ such that

$$
y\left(t, z_{m}\right)<\varepsilon_{y} \text { for } t \geq \tau_{2}^{(m)} .
$$

This leads to

$$
\begin{aligned}
\dot{x}_{1}\left(t, z_{m}\right) \geq & x_{1}\left(t, z_{m}\right)\left[b_{1}(t)-c_{1}(t) \varepsilon_{y}-a_{1}(t) x_{1}\left(t, z_{m}\right)\right] \\
& +D(t)\left(x_{2}\left(t, z_{m}\right)-x_{1}\left(t, z_{m}\right)\right), \\
\dot{x}_{2}\left(t, z_{m}\right)= & x_{2}\left(t, z_{m}\right)\left[b_{2}(t)-a_{2}(t) x_{2}\left(t, z_{m}\right)\right]+D(t)\left(x_{1}\left(t, z_{m}\right)-x_{2}\left(t, z_{m}\right)\right)
\end{aligned}
$$

for $t \geq \tau_{2}^{(m)}$. Let $\left(u_{1}(t), u_{2}(t)\right)$ be any positive solution of the following auxiliary equations:

$$
\begin{aligned}
& \dot{u}_{1}=u_{1}\left[b_{1}(t)-a_{1}(t) u_{1}-c_{1}(t) \varepsilon_{y}\right]+D(t)\left(u_{2}-u_{1}\right), \\
& \dot{u}_{2}=u_{2}\left[b_{2}(t)-a_{2}(t) u_{2}\right]+D(t)\left(u_{1}-u_{2}\right) .
\end{aligned}
$$

By (3.14) and Lemma 2.5, (3.23) has a unique positive and $\omega$-periodic solution $\left(u_{1}^{*}(t), u_{2}^{*}(t)\right)$, which is globally asymptotically stable. So we have

$$
x_{i}\left(t, z_{m}\right)>\frac{u_{i}^{*}(t)}{2}, \quad i=1,2,
$$

for sufficiently large $t>0$ and $m>N_{0}$, which is a contradiction with (3.12). This completes the proof.

Proposition 3.4. There exists positive constants $\gamma_{x}$ such that

$$
\liminf _{t \rightarrow \infty} \rho_{x}(t) \geq \gamma_{x},
$$

where $\rho_{x}(t)=x_{1}(t)+x_{2}(t)$.

Proof. Suppose that (3.25) is not true; then there exists a sequence $\left\{z_{m}\right\} \subset \mathbb{R}_{+}^{3}$ such that

$$
\liminf _{t \rightarrow \infty} \rho_{x}\left(t, z_{m}\right)<\frac{\eta_{x}}{2 m^{2}}, \quad m=1,2, \ldots
$$

On the other hand, by Proposition 3.3, we have

$$
\lim _{t \rightarrow \infty} \sup \rho_{x}\left(t, z_{m}\right) \geq \lim _{t \rightarrow \infty} \sup x_{1}\left(t, z_{m}\right) \geq \eta_{x}, \quad m=1,2, \ldots
$$

Hence there are two time sequences $\left\{s_{\mathcal{q}}^{(m)}\right\}$ and $\left\{t_{\mathcal{q}}^{(m)}\right\}$ satisfying the following conditions:

$$
\begin{gathered}
0<s_{1}^{(m)}<t_{1}^{(m)}<s_{2}^{(m)}<t_{2}^{(m)}<\cdots<s_{q}^{(m)}<t_{q}^{(m)}<\cdots, \quad s_{q}^{(m)} \longrightarrow \infty, t_{q}^{(m)} \longrightarrow \infty(q \longrightarrow \infty), \\
\rho_{x}\left(s_{q}^{(m)}, z_{m}\right)=\frac{\eta_{x}}{m}, \quad \rho_{x}\left(t_{q}^{(m)}, z_{m}\right)=\frac{\eta_{x}}{m^{2}}, \\
\frac{\eta_{x}}{m^{2}}<\rho_{x}\left(t, z_{m}\right)<\frac{\eta_{x}}{m}, \quad t \in\left(s_{q}^{(m)}, t_{q}^{(m)}\right) .
\end{gathered}
$$


By Proposition 3.2, for a given integer $m>0$, there is a $T_{1}^{(m)}>0$ such that

$$
x_{i}\left(t, z_{m}\right) \leq M_{x}, \quad y\left(t, z_{m}\right) \leq M_{y}, \quad \text { for } t \geq T_{1}^{(m)}, i=1,2 .
$$

Because of $s_{q}^{(m)} \rightarrow \infty$ as $q \rightarrow \infty$, there is a positive integer $K^{(m)}$ such that $s_{q}^{(m)}>T_{1}^{(m)}$ as $q \geq K^{(m)}$; hence

$$
\begin{aligned}
& \dot{x}_{1}\left(t, z_{m}\right) \geq x_{1}\left(t, z_{m}\right)\left[b_{1}(t)-a_{1}(t) M_{x}-c_{1}(t) M_{y}\right]+D(t)\left(x_{2}\left(t, z_{m}\right)-x_{1}\left(t, z_{m}\right)\right), \\
& \dot{x}_{2}\left(t, z_{m}\right) \geq x_{2}\left(t, z_{m}\right)\left[b_{2}(t)-a_{2}(t) M_{x}\right]+D(t)\left(x_{1}\left(t, z_{m}\right)-x_{2}\left(t, z_{m}\right)\right)
\end{aligned}
$$

for $q \geq K^{(m)}$; so

$$
\dot{\rho}_{x}\left(t, z_{m}\right) \geq \zeta(t) \rho_{x}\left(t, z_{m}\right)
$$

for $q \geq K^{(m)}, t \in\left[s_{q}^{(m)}, t_{q}^{(m)}\right]$, where $\zeta(t)=\min \left\{b_{1}(t)-a_{1}(t) M_{x}-c_{1}(t) M_{y}, b_{2}(t)-\right.$ $a_{2}(t) M_{x}$ \}. Integrating (3.32) from $s_{q}^{(m)}$ to $t_{q}^{(m)}$ yields

$$
\rho_{x}\left(t_{q}^{(m)}, z_{m}\right) \geq \rho_{x}\left(s_{q}^{(m)}, z_{m}\right) \exp \int_{s_{q}^{(m)}}^{t_{q}^{(m)}} \zeta(t) d t
$$

or

$$
-\int_{s_{q}^{(m)}}^{t_{q}^{(m)}} \zeta(t) d t \geq \ln m \text { for } q \geq K^{(m)} .
$$

If $A_{\omega}(\zeta(t)) \geq 0$, this leads to a contradiction; otherwise, if $A_{\omega}(\zeta(t))<0$, we have

$$
t_{q}^{(m)}-s_{q}^{(m)} \longrightarrow \infty \quad\left(m \longrightarrow \infty, q \geq K^{(m)}\right)
$$

according to the boundedness of $\zeta(t)$. By (3.13) and (3.14), there are constants $P>0$ and $N_{0}>0$ such that

$$
\begin{gathered}
\frac{\eta_{x}}{m}<\varepsilon_{x}, \quad t_{q}^{(m)}-s_{q}^{(m)}>2 P, \\
M_{y} \exp \int_{0}^{P}\left[-d(t)+c_{2}(t) \varepsilon_{x}-l(t) \varepsilon_{y}\right] d t<\varepsilon_{y}, \quad \int_{0}^{a} \phi_{\varepsilon}(t) d t>0
\end{gathered}
$$

for $m \geq N_{0}, q \geq K^{(m)}$, and $a \geq P$. Inequality (3.36) implies

$$
x_{i}\left(t, z_{m}\right)<\varepsilon_{x}, \quad i=1,2, t \in\left[s_{q}^{(m)}, t_{q}^{(m)}\right]
$$

for $m \geq N_{0}, q \geq K^{(m)}$. For the positive $\varepsilon_{y}$ satisfying (3.14) and (3.37), we have the following two circumstances:

(i) $y\left(t, z_{m}\right) \geq \varepsilon_{y}$ for all $t \in\left[s_{q}^{(m)}, s_{q}^{(m)}+P\right]$;

(ii) there exists $\tau_{q 1}^{(m)} \in\left[s_{q}^{(m)}, s_{q}^{(m)}+P\right]$ such that $y\left(\tau_{q 1}^{(m)}, z_{m}\right)<\varepsilon_{y}$. 
If (i) holds, by (3.38) we have

$$
\begin{aligned}
\varepsilon_{y} & \leq y\left(s_{q}^{(m)}+P, z_{m}\right) \\
& \leq y\left(s_{q}^{(m)}, z_{m}\right) \exp \int_{s_{q}^{(m)}}^{s_{q}^{(m)}+P}\left[-d(t)+c_{2}(t) \varepsilon_{x}-l(t) \varepsilon_{y}\right] d t \\
& \leq M_{y} \exp \int_{0}^{P}\left[-d(t)+c_{2}(t) \varepsilon_{x}-l(t) \varepsilon_{y}\right] d t \\
& <\varepsilon_{y},
\end{aligned}
$$

which is a contradiction.

If (ii) holds, we now claim that

$$
y\left(t, z_{m}\right) \leq \varepsilon_{y} \exp (\alpha \omega), \quad t \in\left(\tau_{q 1}^{(m)}, t_{q}^{(m)}\right]
$$

Otherwise, there exists $\tau_{q 2}^{(m)} \in\left(\tau_{q 1}^{(m)}, t_{q}^{(m)}\right]$ such that

$$
y\left(\tau_{q 2}^{(m)}, z_{m}\right)>\varepsilon_{y} \exp (\alpha \omega) .
$$

By the continuity of $y\left(t, z_{m}\right)$, there must exist $\tau_{q 3}^{(m)} \in\left(\tau_{q 1}^{(m)}, \tau_{q 2}^{(m)}\right)$ such that

$$
\begin{gathered}
y\left(\tau_{q 3}^{(m)}, z_{m}\right)=\varepsilon_{y}, \\
y\left(t, z_{m}\right)>\varepsilon_{y} \quad \text { for } t \in\left(\tau_{q 3}^{(m)}, \tau_{q 2}^{(m)}\right) .
\end{gathered}
$$

Denote by $P^{(m)}$ the nonnegative integer such that $\tau_{q 2}^{(m)} \in\left(\tau_{q 3}^{(m)}+P^{(m)} \omega, \tau_{q 3}^{(m)}+\left(P^{(m)}+\right.\right.$ 1) $\omega$ ]. By (3.13), we obtain

$$
\begin{aligned}
\varepsilon_{y} \exp (\alpha \omega) & <y\left(\tau_{q 2}^{(m)}, z_{m}\right) \\
& <y\left(\tau_{q 3}^{(m)}, z_{m}\right) \exp \int_{\tau_{q 3}^{(m)}}^{\tau_{q 2}^{(m)}}\left[-d(t)+c_{2}(t) \varepsilon_{x}-l(t) \varepsilon_{y}\right] d t \\
& =\varepsilon_{y} \exp \left\{\int_{\tau_{q 3}^{(m)}}^{\tau_{q 3}^{(m)}+P^{(m)} \omega}+\int_{\tau_{q 3}^{(m)}+P^{(m)} \omega}^{\tau_{q 2}^{(m)}}\right\}\left[-d(t)+c_{2}(t) \varepsilon_{x}-l(t) \varepsilon_{y}\right] d t \\
& <\varepsilon_{y} \exp (\alpha \omega) .
\end{aligned}
$$

This contradiction establishes that (3.40) is true; particularly, (3.40) holds for $t \in$ $\left[s_{q}^{(m)}+P, t_{q}^{(m)}\right]$. By (3.29) and (3.14), we have

$$
\begin{aligned}
\frac{\eta_{x}}{m^{2}} & =\rho_{x}\left(t_{q}^{(m)}, z_{m}\right) \\
& \geq \rho_{x}\left(s_{q}^{(m)}+P, z_{m}\right) \exp \int_{s_{q}^{(m)}+P}^{t_{q}^{(m)}} \phi_{\varepsilon}(t) d t \\
& >\frac{\eta_{x}}{m^{2}},
\end{aligned}
$$

which is also a contradiction. This completes the proof. 
Proposition 3.5. There exist positive constants $\gamma_{x i}(i=1,2)$ such that

$$
\liminf _{t \rightarrow \infty} x_{i}(t) \geq \gamma_{x i} \quad(i=1,2) .
$$

Proof. Inequality (3.25) implies that there exists $T_{2} \geq T_{1}$ such that

$$
\rho_{x}(t)=x_{1}(t)+x_{2}(t) \geq \gamma_{x} \text { for } t \geq T_{2} \text {. }
$$

Hence,

$$
\begin{aligned}
\dot{x}_{1} & =x_{1}\left[b_{1}(t)-2 D(t)-a_{1}(t) x_{1}-c_{1}(t) y\right]+D(t) \rho_{x}(t) \\
& \geq-a_{1}^{M} x_{1}^{2}+\left(b_{1}^{L}-2 D^{M}-c_{1}^{M} M_{y}\right) x_{1}+D^{L} \gamma_{x}:=F_{1}\left(x_{1}\right), \\
\dot{x}_{2} & \geq-a_{2}^{M} x_{2}^{2}+\left(b_{2}^{L}-2 D^{M}\right) x_{2}+D^{L} \gamma_{x}:=F_{2}\left(x_{2}\right)
\end{aligned}
$$

for $t \geq T_{2}$. The algebraic equation $F_{1}\left(x_{1}\right)=0$ gives us one positive root

$$
\tilde{x}_{1}=\frac{b_{1}^{L}-2 D^{M}-c_{1}^{M} M_{y}+\sqrt{\left(b_{1}^{L}-2 D^{M}-c_{1}^{M} M_{y}\right)^{2}+4 D^{L} a_{1}^{M} \gamma_{x}}}{2 a_{1}^{M}} .
$$

Clearly, $F_{1}\left(x_{1}\right)>0$ for every positive number $x_{1}\left(0<x_{1}<\tilde{x}_{1}\right)$. Choose $\gamma_{x 1}\left(0<\gamma_{x 1}<\right.$ $\left.\tilde{x}_{1}\right),\left.\dot{x}_{1}\right|_{x_{1}=\gamma_{x 1}} \geq F_{1}\left(\gamma_{x 1}\right)>0$. If $x_{1}\left(T_{2}\right) \geq \gamma_{x 1}$, then it also holds for $t \geq T_{2}$; if $x_{1}\left(T_{2}\right)<$ $\gamma_{x 1}$, then

$$
\dot{x}_{1}\left(T_{2}\right) \geq \inf \left\{F_{1}\left(x_{1}\right) \mid 0 \leq x_{1}<\gamma_{x 1}\right\}>0
$$

there exists $T_{3}\left(\geq T_{2}\right)$ such that $x_{1}(t)>\gamma_{x 1}$ for $t \geq T_{3}$.

Similarly, there exists $\gamma_{x 2}>0$ and $T_{4}\left(\geq T_{3}\right)$ such that $x_{2}(t)>\gamma_{x 2}$ for $t \geq T_{4}$. This completes the proof.

Proposition 3.6. Suppose that (3.1) holds. Then there exists a positive constant $\eta_{y}$ such that

$$
\lim _{t \rightarrow \infty} \sup y(t)>\eta_{y}
$$

Proof. By (3.1), we can choose constant $\varepsilon_{0}>0$ such that

$$
A_{\omega}\left(\psi_{\varepsilon_{0}}(t)\right)>0
$$

where

$$
\psi_{\varepsilon_{0}}(t)=-d(t)+c_{2}(t) x_{1}^{*}(t)-c_{2}(t) \varepsilon_{0}-l(t) \varepsilon_{0} .
$$

Consider the following equations with parameter $\alpha\left(0<\alpha<b_{1}^{L} / 2 c_{1}^{M}\right)$ :

$$
\begin{aligned}
& \dot{x}_{1}=x_{1}\left[b_{1}(t)-2 \alpha c_{1}(t)-a_{1}(t) x_{1}\right]+D(t)\left(x_{2}-x_{1}\right), \\
& \dot{x}_{2}=x_{2}\left[b_{2}(t)-a_{2}(t) x_{2}\right]+D(t)\left(x_{1}-x_{2}\right) .
\end{aligned}
$$


By Lemma 2.5, (3.53) has a unique positive $\omega$-periodic solution $\left(x_{1 \alpha}(t), x_{2 \alpha}(t)\right)$ which is globally asymptotically stable. Let $\left(\bar{x}_{1 \alpha}(t), \bar{x}_{2 \alpha}(t)\right)$ be the solution of (3.53) with initial condition $\bar{x}_{i \alpha}(0)=x_{i}^{*}(0), i=1,2$, where $\left(x_{1}^{*}(t), x_{2}^{*}(t)\right)$ is the positive and $\omega$-periodic solution of (2.10); then for the given $\varepsilon_{0}$, there exists $T_{5} \geq T_{4}$ such that

$$
\left|\bar{x}_{1 \alpha}(t)-x_{1 \alpha}(t)\right|<\frac{\varepsilon_{0}}{4} \quad \text { for } t \geq T_{5} .
$$

By the continuity of solution to parameter $\alpha$, we have $\left(\bar{x}_{1 \alpha}(t), \bar{x}_{2 \alpha}(t)\right) \rightarrow\left(x_{1}^{*}(t), x_{2}^{*}(t)\right)$ uniformly in $\left[T_{5}, T_{5}+\omega\right]$ as $\alpha \rightarrow 0$. Hence for $\varepsilon_{0}>0$, there exists positive $\alpha_{0}=\alpha_{0}\left(\varepsilon_{0}\right)<$ $b_{1}^{L} / 2 c_{1}^{M}$ such that

$$
\left|\bar{x}_{1 \alpha}(t)-x_{1}^{*}(t)\right|<\frac{\varepsilon_{0}}{4} \quad \text { for } t \in\left[T_{5}, T_{5}+\omega\right], 0<\alpha<\alpha_{0} .
$$

So we have

$$
\left|x_{1 \alpha}(t)-x_{1}^{*}(t)\right| \leq\left|\bar{x}_{1 \alpha}(t)-x_{1 \alpha}(t)\right|+\left|\bar{x}_{1 \alpha}(t)-x_{1}^{*}(t)\right|<\frac{\varepsilon_{0}}{2}
$$

for $t \in\left[T_{5}, T_{5}+\omega\right]$. Since $x_{1 \alpha}(t)$ and $x_{1}^{*}(t)$ are all $\omega$-periodic, we have

$$
\left|x_{1 \alpha}(t)-x_{1}^{*}(t)\right|<\frac{\varepsilon_{0}}{2} \text { for } t \geq 0,0<\alpha<\alpha_{0} .
$$

Choosing constant $\alpha_{1}\left(0<\alpha_{1}<\alpha_{0}, 2 \alpha_{1}<\varepsilon_{0}\right)$, then

$$
x_{1 \alpha_{1}}(t) \geq x_{1}^{*}(t)-\frac{\varepsilon_{0}}{2}, \quad t \geq 0 .
$$

Suppose that the conclusion (3.50) is not true. Then there exists $Z \in \mathbb{R}_{+}^{3}$ such that for the positive solution $\left(x_{1}(t), x_{2}(t), y(t)\right)$ of (1.4) with initial condition $\left(x_{1}(0), x_{2}(0), y(0)\right)=$ $Z$, we have

$$
\lim _{t \rightarrow \infty} \sup y(t)<\alpha_{1}
$$

So there exists $T_{6} \geq T_{5}$ such that

$$
y(t)<2 \alpha_{1} \text { for } t \geq T_{6}
$$

and hence,

$$
\begin{aligned}
& \dot{x}_{1} \geq x_{1}\left[b_{1}(t)-2 \alpha_{1} c_{1}(t)-a_{1}(t) x_{1}\right]+D(t)\left(x_{2}-x_{1}\right), \\
& \dot{x}_{2}=x_{2}\left[b_{2}(t)-a_{2}(t) x_{2}\right]+D(t)\left(x_{1}-x_{2}\right) .
\end{aligned}
$$

Let $\left(u_{1}(t), u_{2}(t)\right)$ be the solution of (3.53) with $\alpha=\alpha_{1}$ and $u_{i}\left(T_{6}\right)=x_{i}\left(T_{6}\right), i=1,2$. By Lemma 2.3, we know that

$$
x_{i}(t) \geq u_{i}(t), \quad t \geq T_{6}, i=1,2 .
$$

By the globally asymptotically stability of $\left(x_{1 \alpha_{1}}(t), x_{2 \alpha_{1}}(t)\right)$, for given $\varepsilon=\varepsilon_{0} / 2$, there exists $T_{7} \geq T_{6}$ such that

$$
\left|u_{1}(t)-x_{1 \alpha_{1}}(t)\right|<\frac{\varepsilon_{0}}{2} \quad \text { for } t \geq T_{7}
$$


So we have

$$
x_{1}(t) \geq u_{1}(t)>x_{1 \alpha_{1}}(t)-\frac{\varepsilon_{0}}{2}, \quad t \geq T_{7},
$$

and hence

$$
x_{1}(t)>x_{1}^{*}(t)-\varepsilon_{0}, \quad t \geq T_{7} .
$$

This implies

$$
\dot{y}(t) \geq \psi_{\varepsilon_{0}}(t) y(t), \quad t \geq T_{7} \text {. }
$$

Integrating the above inequality from $T_{7}$ to $t$ yields

$$
y(t) \geq y\left(T_{7}\right) \exp \int_{T_{7}}^{t} \psi_{\varepsilon_{0}}(t) d t .
$$

By (3.51), we know that $y(t) \rightarrow \infty$ as $t \rightarrow \infty$, which is a contradiction. This completes the proof.

Proposition 3.7. Under the assumption (3.1), there exists a positive constant $\gamma$ y such that

$$
\lim _{t \rightarrow \infty} \inf y(t) \geq \gamma y
$$

Proof. Otherwise, there must exist a sequence $\left\{z_{m}\right\} \subset \mathbb{R}_{+}^{3}$ such that

$$
\lim _{t \rightarrow \infty} \inf y\left(t, z_{m}\right)<\frac{\eta_{y}}{(m+1)^{2}}, \quad m=1,2, \ldots
$$

But

$$
\lim _{t \rightarrow \infty} \sup y\left(t, z_{m}\right)>\eta_{y}, \quad m=1,2, \ldots
$$

according to Proposition 3.6. Hence there are two time sequences $\left\{s_{q}^{(m)}\right\}$ and $\left\{t_{q}^{(m)}\right\}$ satisfying the following conditions:

$$
\begin{gathered}
0<s_{1}^{(m)}<t_{1}^{(m)}<s_{2}^{(m)}<t_{2}^{(m)}<\cdots<s_{q}^{(m)}<t_{q}^{(m)}<\cdots, \\
s_{q}^{(m)} \longrightarrow \infty, \quad t_{q}^{(m)} \longrightarrow \infty \text { as } q \longrightarrow \infty, \\
y\left(s_{q}^{(m)}, z_{m}\right)=\frac{\eta_{y}}{m+1}, \quad y\left(t_{q}^{(m)}, z_{m}\right)=\frac{\eta_{y}}{(m+1)^{2}}, \\
\frac{\eta_{y}}{(m+1)^{2}}<y\left(t, z_{m}\right)<\frac{\eta_{y}}{m+1}, \quad t \in\left(s_{q}^{(m)}, t_{q}^{(m)}\right) .
\end{gathered}
$$

By Proposition 3.2, for a given integer $m>0$, there is a $T_{1}^{(m)}>0$ such that

$$
y\left(t, z_{m}\right) \leq M_{y} \text { for } t \geq T_{1}^{(m)} .
$$


Because $s_{q}^{(m)} \rightarrow \infty$ as $q \rightarrow \infty$, there is a positive integer $K^{(m)}$ such that $s_{q}^{(m)}>T_{1}^{(m)}$ as $q \geq K^{(m)}$; hence

$$
\dot{y}\left(t, z_{m}\right) \geq y\left(t, z_{m}\right)\left[-d(t)-l(t) M_{y}\right]
$$

for $q \geq K^{(m)}, t \in\left[s_{\mathcal{q}}^{(m)}, t_{q}^{(m)}\right]$. Integrating the above inequality from $s_{\mathcal{q}}^{(m)}$ to $t_{q}^{(m)}$, we get

$$
y\left(t_{q}^{(m)}, z_{m}\right) \geq y\left(s_{q}^{(m)}, z_{m}\right) \exp \int_{s_{q}^{(m)}}^{t_{q}^{(m)}}\left[-d(t)-l(t) M_{y}\right] d t .
$$

So we have

$$
\int_{s_{q}^{(m)}}^{t_{q}^{(m)}}\left[d(t)+l(t) M_{y}\right] d t \geq \ln (m+1) \quad \text { for } q \geq K^{(m)} .
$$

According to the boundedness of the function $d(t)+l(t) M_{y}$, we know that

$$
t_{q}^{(m)}-s_{q}^{(m)} \longrightarrow \infty \quad \text { as } m \longrightarrow \infty, q \geq K^{(m)} .
$$

By (3.51), there are constants $P>0$ and an integer $N_{0}>0$ such that

$$
\begin{gathered}
\frac{\eta_{y}}{m+1}<\alpha_{1}<\varepsilon_{0}, \quad t_{q}^{(m)}-s_{q}^{(m)}>2 P, \\
\int_{0}^{a} \psi_{\varepsilon_{0}}(t) d t>0
\end{gathered}
$$

for $m \geq N_{0}, q \geq K^{(m)}$, and $a \geq P$. Further, we have

$$
y\left(t, z_{m}\right)<\alpha_{1}, \quad t \in\left[s_{q}^{(m)}, t_{q}^{(m)}\right]
$$

for $m \geq N_{0}, q \geq K^{(m)}$. In addition, for $t \in\left[s_{q}^{(m)}, t_{q}^{(m)}\right]$, we have

$$
\begin{aligned}
\dot{x}_{1}\left(t, z_{m}\right) \geq & x_{1}\left(t, z_{m}\right)\left[b_{1}(t)-2 \alpha_{1} c_{1}(t)-a_{1}(t) x_{1}\left(t, z_{m}\right)\right] \\
& +D(t)\left(x_{2}\left(t, z_{m}\right)-x_{1}\left(t, z_{m}\right)\right), \\
\dot{x}_{2}\left(t, z_{m}\right)= & x_{2}\left(t, z_{m}\right)\left[b_{2}(t)-a_{2}(t) x_{2}\left(t, z_{m}\right)\right]+D(t)\left(x_{1}\left(t, z_{m}\right)-x_{2}\left(t, z_{m}\right)\right) .
\end{aligned}
$$

Let $\left(u_{1}(t), u_{2}(t)\right)$ be the solution of (3.53) with $\alpha=\alpha_{1}$ and $u_{i}\left(s_{q}^{(m)}\right)=x_{i}\left(s_{q}^{(m)}, z_{m}\right)$. By Lemma 2.3, we have

$$
x_{i}\left(t, z_{m}\right) \geq u_{i}(t), \quad t \in\left[s_{q}^{(m)}, t_{q}^{(m)}\right]
$$

Further, by Propositions 3.2, 3.5, and $s_{q}^{(m)} \rightarrow \infty$ as $q \rightarrow \infty$, we can choose $K_{1}^{(m)}>K^{(m)}$ such that

$$
\gamma_{x i} \leq x_{i}\left(s_{q}^{(m)}, z_{m}\right) \leq M_{x}, \quad i=1,2
$$

for $q \geq K_{1}^{(m)}$. For $\alpha=\alpha_{1}$, (3.53) has a unique positive $\omega$-periodic solution $\left(x_{1 \alpha_{1}}(t)\right.$, $\left.x_{2 \alpha_{1}}(t)\right)$ which is globally asymptotically stable. In addition, by the periodicity of (3.53), 
the periodic solution $\left(x_{1 \alpha_{1}}(t), x_{2 \alpha_{1}}(t)\right)$ is uniformly asymptotically stable with respect to the compact set $\Omega=\left\{\left(x_{1}, x_{2}\right): \gamma_{x i} \leq x_{i} \leq M_{x}, i=1,2\right\}$. Hence, for the given $\varepsilon_{0}$ in Proposition 3.6, there exists $T_{0}(>P)$, which is independent of $m$ and $q$, such that

$$
u_{1}(t) \geq x_{1 \alpha_{1}}(t)-\frac{\varepsilon_{0}}{2}, \quad t \geq T_{0}+s_{q}^{(m)} .
$$

Combining (3.58), we have

$$
u_{1}(t) \geq x_{1}^{*}(t)-\varepsilon_{0} \quad \text { for } t \geq T_{0}+s_{q}^{(m)} .
$$

From (3.76), there exists a positive integer $N_{1} \geq N_{0}$ such that $t_{q}^{(m)}>s_{q}^{(m)}+2 T_{0}>s_{q}^{(m)}+$ $2 P$ for $m \geq N_{1}$ and $q \geq K_{1}^{(m)}$. So we have

$$
x_{1}\left(t, z_{m}\right) \geq x_{1}^{*}(t)-\varepsilon_{0}, \quad t \in\left[s_{q}^{(m)}+T_{0}, t_{q}^{(m)}\right]
$$

as $m \geq N_{1}$ and $q \geq K_{1}^{(m)}$. Hence,

$$
\dot{y}\left(t, z_{m}\right) \geq \psi_{\varepsilon_{0}}(t) y\left(t, z_{m}\right)
$$

for $t \in\left[s_{q}^{(m)}+T_{0}, t_{q}^{(m)}\right]$. Integrating the above inequality from $s_{q}^{(m)}+T_{0}$ to $t_{q}^{(m)}$ yields

$$
y\left(t_{q}^{(m)}, z_{m}\right) \geq y\left(s_{q}^{(m)}+T_{0}, z_{m}\right) \exp \int_{s_{q}^{(m)}+T_{0}}^{t_{q}^{(m)}} \psi_{\varepsilon_{0}}(t) d t,
$$

that is to say,

$$
\frac{\eta_{y}}{(m+1)^{2}} \geq \frac{\eta_{y}}{(m+1)^{2}} \exp \int_{s_{q}^{(m)}+T_{0}}^{t_{q}^{(m)}} \psi_{\varepsilon_{0}}(t) d t>\frac{\eta_{y}}{(m+1)^{2}},
$$

which is a contradiction. This completes the proof.

Combining Propositions 3.2 to 3.6, we complete the proof of the sufficiency of Theorem 3.1.

To prove the necessity of Theorem 3.1, we will show that

$$
\lim _{t \rightarrow \infty} y(t)=0
$$

under the following condition:

$$
A_{\omega}\left[-d(t)+c_{2}(t) x_{1}^{*}(t)\right] \leq 0 .
$$

In fact, by (3.89), we know that for every given $\varepsilon(0<\varepsilon<1)$, there exists $\varepsilon_{1}>0$ and $\varepsilon_{0}>0$ such that

$$
A_{\omega}\left[-d(t)+c_{2}(t)\left(x_{1}^{*}(t)+\varepsilon_{1}\right)-l(t) \varepsilon\right] \leq \varepsilon_{1} A_{\omega}\left(c_{2}(t)\right)-\varepsilon A_{\omega}(l(t)) \leq-\varepsilon_{0} .
$$

Since

$$
\begin{aligned}
& \dot{x}_{1} \leq x_{1}\left[b_{1}(t)-a_{1}(t) x_{1}\right]+D(t)\left(x_{2}-x_{1}\right), \\
& \dot{x}_{2}=x_{2}\left[b_{2}(t)-a_{2}(t) x_{2}\right]+D(t)\left(x_{1}-x_{1}\right),
\end{aligned}
$$


we know that for the given $\varepsilon_{1}$, there exists $T^{(1)}>0$ such that

$$
x_{1}(t) \leq x_{1}^{*}(t)+\varepsilon_{1} \quad \text { for } t \geq T^{(1)} .
$$

By (3.90), we have

$$
A_{\omega}\left[-d(t)+c_{2}(t) x_{1}(t)-l(t) \varepsilon\right] \leq-\varepsilon_{0}
$$

for $t \geq T^{(1)}$. Firstly, there must exist $T^{(2)}$ such that $y\left(T^{(2)}\right)<\varepsilon$. Otherwise, we have

$$
\varepsilon \leq y(t) \leq y\left(T^{(1)}\right) \exp \int_{T^{(1)}}^{t}\left[-d(s)+c_{2}(s) x_{1}(s)-q(s) \varepsilon\right] d s \longrightarrow 0 \quad \text { as } t \rightarrow \infty .
$$

This implies $\varepsilon \leq 0$, which is a contradiction. Let $M(\varepsilon)=\max _{0 \leq t \leq \omega}\left\{d(t)+c_{2}(t) x_{1}(t)+\right.$ $l(t) \varepsilon$. By Proposition 3.2, we know that $x_{1}(t)$ is bounded. So $M(\varepsilon)$ is also bounded for $\varepsilon \in[0,1]$.

Secondly, we have

$$
y(t) \leq \varepsilon \exp (M(\varepsilon) \omega) \text { for } t \geq T^{(2)} .
$$

Otherwise, there exists $T^{(3)}>T^{(2)}$ such that

$$
y\left(T^{(3)}\right)>\varepsilon \exp (M(\varepsilon) \omega) .
$$

By the continuity of $y(t)$, there must exist $T^{(4)} \in\left(T^{(2)}, T^{(3)}\right)$ such that $y\left(T^{(4)}\right)=\varepsilon$ and $y(t)>\varepsilon$ for $t \in\left(T^{(4)}, T^{(3)}\right]$. Let $P_{1}$ be the nonnegative integer such that $T^{(3)} \in$ $\left(T^{(4)}+P_{1} \omega, T^{(4)}+\left(P_{1}+1\right) \omega\right]$. By (3.93), we have

$$
\begin{aligned}
\varepsilon \exp (M(\varepsilon) \omega) & <y\left(T^{(3)}\right) \\
& <y\left(T^{(4)}\right) \exp \int_{T^{(4)}}^{T^{(3)}}\left[-d(t)+c_{2}(t) x_{1}(t)-l(t) \varepsilon\right] d t \\
& =\varepsilon \exp \left\{\int_{T^{(4)}}^{T^{(4)}+P_{1} \omega}+\int_{T^{(4)}+P_{1} \omega}^{T^{(3)}}\right\}\left[-d(t)+c_{2}(t) x_{1}(t)-l(t) \varepsilon\right] d t \\
& <\varepsilon \exp (M(\varepsilon) \omega),
\end{aligned}
$$

which is a contradiction. This implies that (3.95) holds. Further by the arbitrariness of $\varepsilon$, we know that $y(t) \rightarrow 0$ as $t \rightarrow \infty$. This completes the proof.

Applying Theorem 3.1 to autonomous system (1.1) directly, we have the following corollary.

COROLLARY 3.8. System (1.1) is permanent if and only if (1.2) holds.

This corollary implies that the strong persistence is equivalent to the permanence for system (1.1), and hence improves the main result (cf. [15, Theorem 2]). 
REMARK 3.9. In [18], Song and Chen obtained that if the following conditions

$$
\begin{aligned}
& N_{2}^{*}:=\frac{c_{2}^{M}}{l^{L}} \max \left\{\frac{b_{1}^{M}}{a_{1}^{L}}, \frac{b_{2}^{M}}{a_{2}^{L}}\right\}<\frac{b_{1}^{L}}{c_{1}^{M}}, \\
& m^{*}:=\min \left\{\frac{b_{1}^{L}-c_{1}^{M} N_{2}^{*}}{a_{1}^{M}}, \frac{b_{2}^{L}}{a_{2}^{M}}\right\}>\frac{d^{M}}{c_{2}^{L}},
\end{aligned}
$$

or

$$
-d^{M}+c_{2}^{L} m^{*}>0
$$

holds, then system (1.4) is permanent. According to [18, Theorem 1], we have $m^{*} \leq$ $x^{*}(t)$, and $A_{\omega}\left(-d(t)+c_{2}(t) x_{1}^{*}(t)\right) \geq-d^{M}+c_{2}^{L} m^{*}$. Hence (3.100) implies (3.1). Further, we give an example where condition (3.1) holds, but conditions (3.98) and (3.100) do not hold.

EXAMPLE 3.10. We consider the model

$$
\begin{aligned}
& \dot{x}_{1}=x_{1}\left[1+\frac{1}{2} \sin t-x_{1}-y\right]+\frac{1}{2}\left(x_{2}-x_{1}\right), \\
& \dot{x}_{2}=x_{2}\left[1-\frac{1}{2} \sin t-x_{2}\right]+\frac{1}{2}\left(x_{1}-x_{2}\right), \\
& \dot{y}=y\left[-d_{0}+\frac{1}{2} x_{1}-y\right]
\end{aligned}
$$

where $d_{0}$ is a positive number. By simple calculation, we have

$$
N_{2}^{*}=\frac{3}{4}, \quad m^{*}=-\frac{1}{4}<0
$$

Hence (3.100) does not hold. We cannot get the permanence of (3.101) from the results of Song and Chen [18]. However, we can obtain its permanence according to our result. In fact, from Lemma 2.5, we know that the following system, without a predator,

$$
\begin{aligned}
& \dot{x}_{1}=x_{1}\left[1+\frac{1}{2} \sin t-x_{1}\right]+\frac{1}{2}\left(x_{2}-x_{1}\right), \\
& \dot{x}_{2}=x_{2}\left[1-\frac{1}{2} \sin t-x_{2}\right]+\frac{1}{2}\left(x_{1}-x_{2}\right)
\end{aligned}
$$

has a positive periodic solution $\left(x_{1}^{*}(t), x_{2}^{*}(t)\right)$ which is globally asymptotically stable. Denote $(2 \pi)^{-1} \int_{0}^{2 \pi} x_{1}^{*}(t) d t=l_{0}$. Then $l_{0}$ is positive and condition (3.1) holds for $d_{0}<$ $(1 / 2) l_{0}$. The permanence of (3.12) follows from Theorem 3.1, provided $d_{0}<(1 / 2) l_{0}$.

REMARK 3.11. Xu, Chaplain, and Davidson studied a more general model than (1.4) (see [22]) and provided the existence, uniqueness, and global stability of periodic solutions of the more general periodic predator-prey system. Conditions for uniform persistence are also stated. We note that their condition (H5) in [22] does not hold for a weak 
patchy environment (see [6]) in the sense that the intrinsic growth rate $b_{i}(t)$ may become negative on some time intervals. However, the discussion in this paper can be used to study the more reasonable weak patchy environment which is important for conservation of some endangered and rare species.

ACKNOWLEDGMENT. The authors thank the referees for their invaluable comments. This work is supported by China National "211" Key project, Jiangsu Provincial Department of Education (02KJB110004), and the National Natural Science Foundation of China.

\section{REFERENCES}

[1] S. Ahmad and A. C. Lazer, Average conditions for global asymptotic stability in a nonautonomous Lotka-Volterra system, Nonlinear Anal. Ser. A: Theory Methods 40 (2000), no. 1-8, 37-49.

[2] L. J. S. Allen, Persistence and extinction in Lotka-Volterra reaction-diffusion equations, Math. Biosci. 65 (1983), no. 1, 1-12.

[3] E. Beretta, F. Solimano, and Y. Takeuchi, Global stability and periodic orbits for two-patch predator-prey diffusion-delay models, Math. Biosci. 85 (1987), no. 2, 153-183.

[4] E. Beretta and Y. Takeuchi, Global asymptotic stability of Lotka-Volterra diffusion models with continuous time delay, SIAM J. Appl. Math. 48 (1988), no. 3, 627-651.

[5] W. C. Chewning Jr., Migratory effects in predator-prey models, Math. Biosci. 23 (1975), 253262.

[6] J. Cui and L. Chen, The effect of diffusion on the time varying logistic population growth, Comput. Math. Appl. 36 (1998), no. 3, 1-9.

[7] H. I. Freedman and Y. Takeuchi, Global stability and predator dynamics in a model of prey dispersal in a patchy environment, Nonlinear Anal. 13 (1989), no. 8, 993-1002.

[8] _ Predator survival versus extinction as a function of dispersal in a predator-prey model with patchy environment, Appl. Anal. 31 (1989), no. 4, 247-266.

[9] H. I. Freedman and P. Waltman, Mathematical models of population interactions with dispersal. I. Stability of two habitats with and without a predator, SIAM J. Appl. Math. 32 (1977), no. 3, 631-648.

[10] M. P. Hassell, The Dynamics of Arthropod Predator-Prey Systems, Monographs in Population Biology, vol. 13, Princeton University Press, New Jersey, 1978.

[11] A. Hastings, Spatial heterogeneity and the stability of predator-prey systems, Theoret. Population Biology 12 (1977), no. 1, 37-48.

[12] R. D. Holt, Population dynamics in two-patch environments: some anomalous consequences of an optimal habitat distribution, Theoret. Population Biol. 28 (1985), no. 2, 181208.

[13] Y. Kuang and Y. Takeuchi, Predator-prey dynamics in models of prey dispersal in two-patch environments, Math. Biosci. 120 (1994), no. 1, 77-98.

[14] S. A. Levin, Dispersion and population interactions, Amer. Natur. 108 (1974), 207-228.

[15] M. C. Luo and Z. E. Ma, Persistence of a two-species Lotka-Volterra model with separation and diffusion, J. Biomath. 12 (1997), no. 1, 52-59 (Chinese).

[16] R. Mahbuba and L. Chen, On the nonautonomous Lotka-Volterra competition system with diffusion, Differential Equations Dynam. Systems 2 (1994), no. 3, 243-253.

[17] H. L. Smith, Cooperative systems of differential equations with concave nonlinearities, Nonlinear Anal. 10 (1986), no. 10, 1037-1052.

[18] X. Song and L. Chen, Persistence and periodic orbits for two-species predator-prey system with diffusion, Canad. Appl. Math. Quart. 6 (1998), no. 3, 233-244.

[19] Y. Takeuchi, Diffusion effect on stability of Lotka-Volterra models, Bull. Math. Biol. 48 (1986), no. 5-6, 585-601. 
[20] _ Global stability in generalized Lotka-Volterra diffusion systems, J. Math. Anal. Appl. 116 (1986), no. 1, 209-221.

[21] Z. Teng, Uniform persistence of the periodic predator-prey Lotka-Volterra systems, Appl. Anal. 72 (1999), no. 3-4, 339-352.

[22] R. Xu, M. A. J. Chaplain, and F. A. Davidson, Periodic solutions for a delayed predator-prey model of prey dispersal in two-patch environments, Nonlinear Anal. Real World Appl. 5 (2004), no. 1, 183-206.

[23] R. Xu and L. Chen, Persistence and global stability for a delayed nonautonomous predatorprey system without dominating instantaneous negative feedback, J. Math. Anal. Appl. 262 (2001), no. 1, 50-61.

[24]__ Global stability for a generalized predator-prey system with time delay in two-patch environments, Appl. Anal. 82 (2003), no. 7, 677-687.

[25] X. Q. Zhao, The qualitative analysis of $n$-species Lotka-Volterra periodic competition systems, Math. Comput. Modelling 15 (1991), no. 11, 3-8.

Jingan Cui: Department of Mathematics, Nanjing Normal University, Nanjing 210097, China

E-mail address: cui ja@pine.njnu.edu.cn

Xinyu Song: Department of Mathematics, Xinyang Teachers College, Xinyang, Henan 464000, China

E-mail address: xysong@xytc.edu.cn 


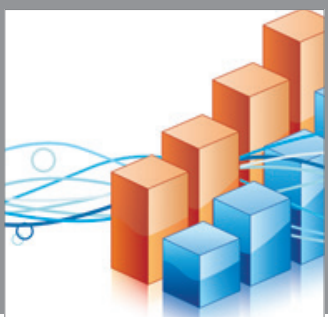

Advances in

Operations Research

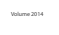

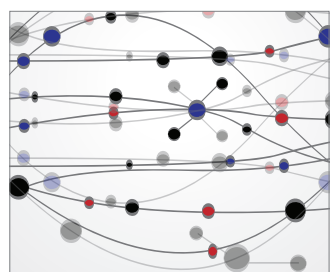

\section{The Scientific} World Journal
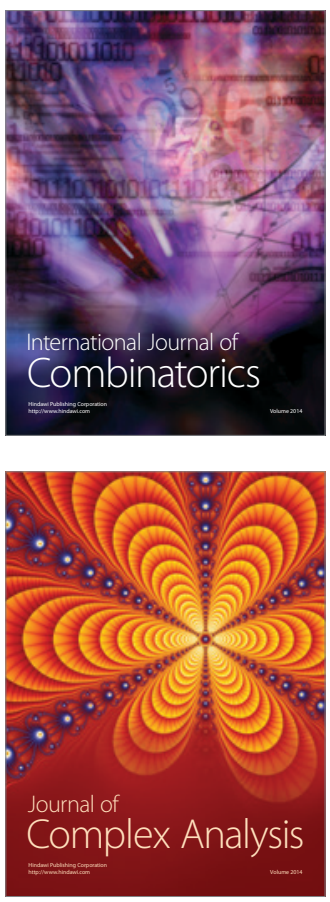

International Journal of

Mathematics and

Mathematical

Sciences
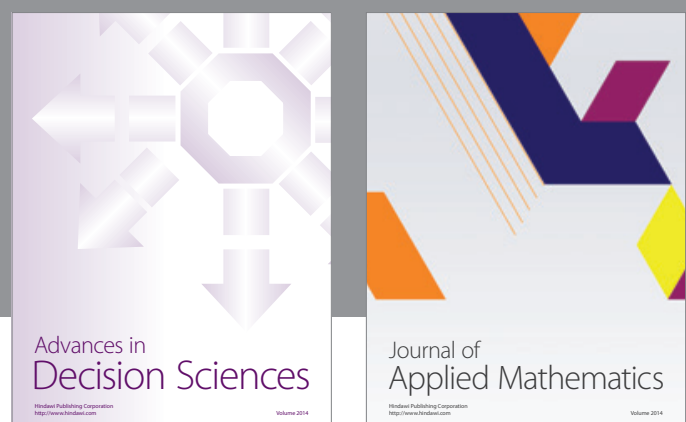

Journal of

Applied Mathematics
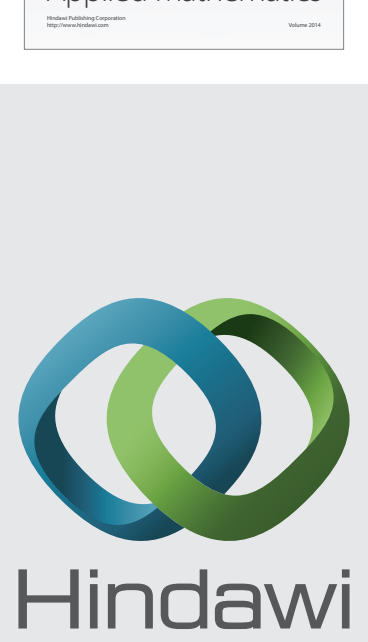

Submit your manuscripts at http://www.hindawi.com
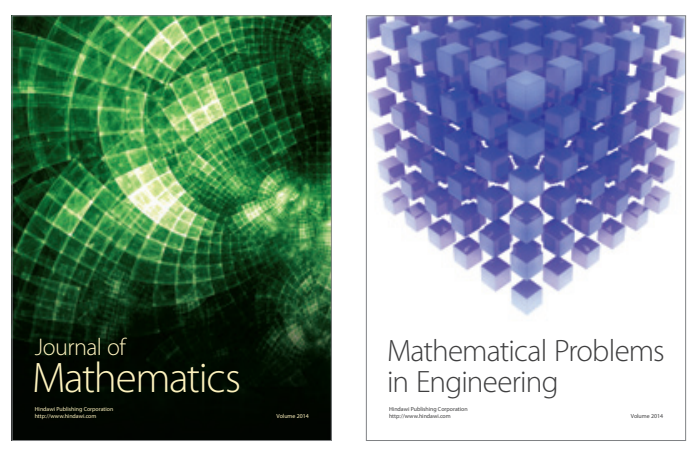

Mathematical Problems in Engineering
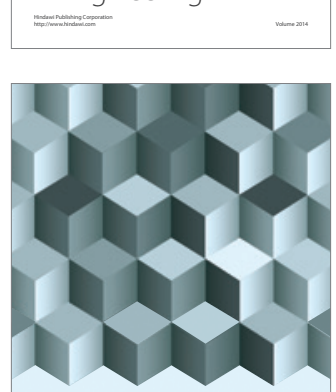

Journal of

Function Spaces
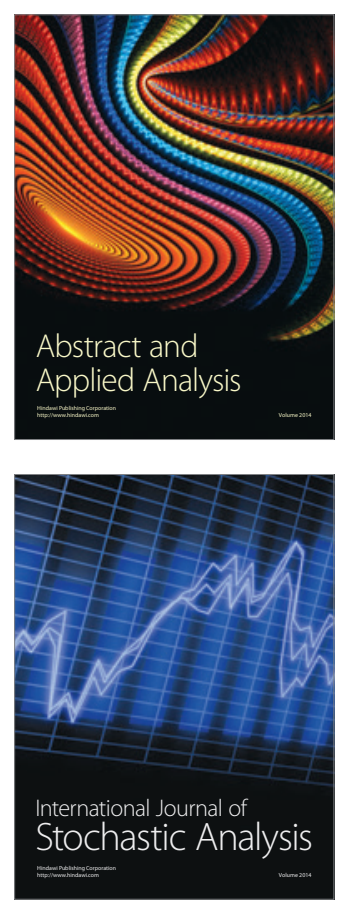

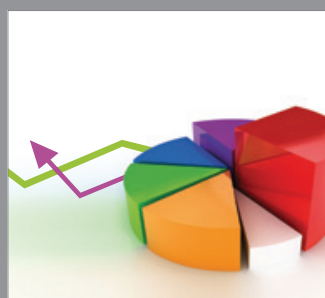

ournal of

Probability and Statistics

Promensencen
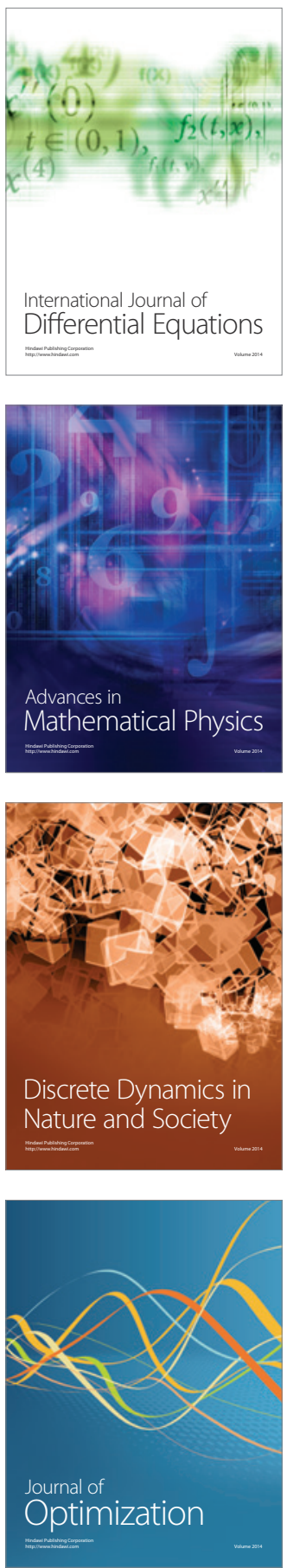